\title{
On the Predictive Ability of Geomagnetic Disturbances from Solar Wind Measurements at Separated Solar Longitude
}

\author{
Wataru Miyake ${ }^{1}$, Tsutomu Nagatsuma ${ }^{2}$ \\ ${ }^{1}$ Department of Aeronautics and Astronautics, Tokai University, Kanagawa, Japan \\ ${ }^{2}$ National Institute of Information and Communications Technology, Tokyo, Japan \\ Email: wmiyake@keyaki.cc.u-tokai.ac.jp
}

Received February 8, 2012; revised February 20, 2012; accepted March 26, 2012

\begin{abstract}
In-situ solar wind measurement at a solar longitude separated from the earth in interplanetary space is expected to provide a great progress in practical space weather forecast, which has been confirmed by some recent studies. We introduce geoeffective solar wind conditions in correlation analysis between STEREO and ACE measurements. We sort solar wind data of ACE by using geomagnetic condition, and evaluate actual ability for predicting geoeffective solar wind arrival at ACE from STEREO-A and B solar wind measurement, by assuming simple corotating structures in interplanetary space. The results show that geomagnetic disturbances are more difficult to be predicted than quiet intervals, suggesting that the simple correlation method of solar wind measurement at separated solar longitude is not enough for accurately predicting geomagnetic disturbances, even though the correlation seems generally high. Although in-situ solar wind monitoring at a vantage point trailing behind the earth would definitely improve the prediction capability of solar wind structure arriving at the terrestrial plasma environment, we emphasize that the predictive ability of geoeffective disturbances would still remain low. We suggest that more sophisticated prediction schemes should be developed.
\end{abstract}

Keywords: Solar Wind; Geomagnetic Disturbances; Space Weather Forecast

\section{Introduction}

The disturbed solar wind impact on terrestrial magnetosphere leading to geomagnetic storms does not only originate from transient phenomena such as coronal mass ejections (CMEs), but also from high-speed solar wind streams emanating from coronal holes. Corotating highspeed streams are known as the source of recurrent geomagnetic storms which tend to be middle-class disturbances, whereas severe geomagnetic storms are generally caused by CMEs [1]. However, impact of the recurrent disturbances is not less important than those of CMEs origin. The flux of relativistic electrons at geostationary orbit, which sometimes damage GEO satellite system, is better correlated with geomagnetic storms due to highspeed streams [2].

Arrival of corotating high-speed streams may be predictable because they are recurrent. The prediction has leading time of almost 27 days (i.e., the rotation period of the sun), which seems magnificent. However, its accuracy is not so impressive. Temporal variation of corotating solar wind structures during 27 days is not negligible. A way of improving accuracy for predicting geomagnetic disturbances due to corotating high-speed streams is to deploy a solar wind monitor at separated solar longitude from the earth. A vantage location for the purpose, for example, is the L5 Lagrange point of the sun-earth system [3-5]. The L5 point, the sun and the earth compose a huge regular triangle in the ecliptic plane. The L5 point is about at solar longitude of 60 degree eastward from the earth, and thus "upstream" with respect to the solar rotation. By placing a solar wind monitor at the L5 point, corotating solar wind streams can be observed in situ about 4.5 days prior to their arrival at the earth.

The solar wind measurement at the L5 point also provides information on background plasma and field structure between the sun and the earth, which is essential for modeling interplanetary transients heading towards the earth. The transients like CMEs are accelerated or decelerated through interaction with the background solar wind (e.g., [6]). The characteristics of the sheath region formed by compressed background solar wind in front of the fast transients are important for development of a geomagnetic storm [7]. In situ measurement of the solar wind at separated solar longitude is expected to provide a 
great progress in practical space weather forecast.

Some recent studies have correlated solar wind measurement at separated solar longitude and discussed possible applications for space weather forecast $[8,9]$, including predictive ability of solar wind monitoring at the L5 point [10,11]. Miyake et al. [10] made a correlation study using data from Nozomi heading toward Mars, and ACE stationed at the L1 point, when they were separated by greater than 50 degrees heliographic longitude. Simunac et al. [11] correlated STEREO-A and B solar wind observations $[12,13]$ when their longitude separation reached about 60 degrees. They reported, in general, a good correlation and remarked its usefulness in forecasting geomagnetic disturbances due to corotating structures in the solar wind.

However, the previous studies never took into account geoeffective solar wind conditions in the correlation analysis and discussion on its application to predicting geomagnetic activities. It may not be so useful to obtain a high correlation only for geomagnetically quiet intervals. What is more needed to predict is the occurrence and magnitude of geomagnetic disturbances. It is well known that geomagnetic disturbances cause various space weather hazards, ranging from satellite system to ground facilities, such as induction current, drastic variation of radiation belt particle flux, heating and expansion of polar upper atmosphere, and development of ionospheric storms. All these phenomena have been a subject under intensive space weather research and are worth being predicted accurately in practical space weather forecast.

In this paper, we introduce geoeffective solar wind conditions in correlation analysis between STEREO and ACE solar wind measurements [14,15]. In Section 2, we describe how to use solar wind data from ACE, STEREO-A and B and how to sort the solar wind data by geomagnetic condition: Kp index (see, for example, [16]). We then try a prediction of the ACE solar wind data from STEREO-A and B solar wind measurements in Section 3. We then evaluate actual ability for predicting geomagnetic disturbances from solar wind measurement at a separate solar longitude in Section 4.

\section{Data and Method}

Our analysis period is from late half of 2007 through early half of 2009. Figure 1 shows histograms of 1-hour averaged solar wind velocity $\mathrm{V}$ (four upper panels) and magnitude of interplanetary magnetic field $|\mathrm{B}|$ (four lower panels) provided from the three spacecraft. We use these 1-hour averaged solar wind parameters throughout the paper. The entire analysis period is divided into four half-year intervals as indicated with DOY (Day Of Year) in the title of each panel. We use the four half-year intervals in the following analysis.

The velocity has decreased in later period. The very slow solar wind in the prolonged solar minimum was already reported $[17,18]$. There is no significant differrence in solar wind velocity among the spacecraft. $|\mathrm{B}|$ is, however, slightly larger at STEREO-A than at STEREO-B, probably due to the difference in radial distance from the sun. The average magnitude is shown in the parentheses. There is no large systematic difference among the three spacecraft data and we use them without any correction.

For the purpose of correlating the data of two measurements, we need to consider the time lag due to the difference in radial distance from the sun and solar longitude. Assuming that the solar wind velocity is constant for the radial expansion, the time lag is calculated by the equation $\mathrm{T}=\left(\mathrm{R}_{\mathrm{s}}-\mathrm{R}_{\mathrm{a}}\right) / \mathrm{Vsw}+\left(\varphi_{s}-\varphi_{a}\right) / \omega$, where $\mathrm{R}_{\mathrm{s}}$ and $\mathrm{R}_{\mathrm{a}}$ are the heliocentric distance of STEREO and ACE, Vsw is the measured solar wind velocity, $\varphi_{s}$ and $\varphi_{a}$ are the solar longitude of STEREO and ACE, and $\omega$ is the angular velocity of the solar rotation, respectively. We neglect the effect of difference in solar latitude between the two spacecraft in this analysis, though large latitudinal gradient of the solar wind velocity have been sometimes reported at the boundaries between the high- and low-speed streams (e.g., [19,20]). The assumption employed here has been widely introduced, and we try a simple prediction scheme of corotating structures for the first step.

Figure 2 summarizes difference in solar wind velocity between STEREO and ACE measurements after the time lag correction. The average of the difference magnitude is plotted as a function of solar longitude separation between the two spacecraft. The left and right panels show the difference between STEREO-B and ACE measurements and between STEREO-A and ACE measurements, respectively. The difference is, in general, increased with the longitude separation, suggesting that larger lag time allows more influence of possible temporal variations in the solar wind.

STEREO-A and B are located in the "downstream" and "upstream" of the earth with respect to the solar rotation, respectively. In this regard, only STEREO-B can be used to measure the solar wind parameters ahead of the earth. We, however, use STEREO-A solar wind measurement by means of inverse time lag. Comparison of the predictions from the STEREO-A and B measurements suggests radial and latitudinal effect, temporal evolution such as transient phenomena, which we neglect in the analysis.

We use Kp index for a geomagnetic parameter, the solar wind velocity $\mathrm{V}$, magnitude of interplanetary magnetic field $|\mathrm{B}|$, and upper limit of possible magnitude of solar wind electric field $\mathrm{V} \cdot|\mathrm{B}|$ for solar wind parameters as correlated with $\mathrm{Kp}$. Since geomagnetic activities are mainly driven by magnetic reconnection between antiparallel magnetic fields, north-south component of interplanetary magnetic field is the most important parameter 

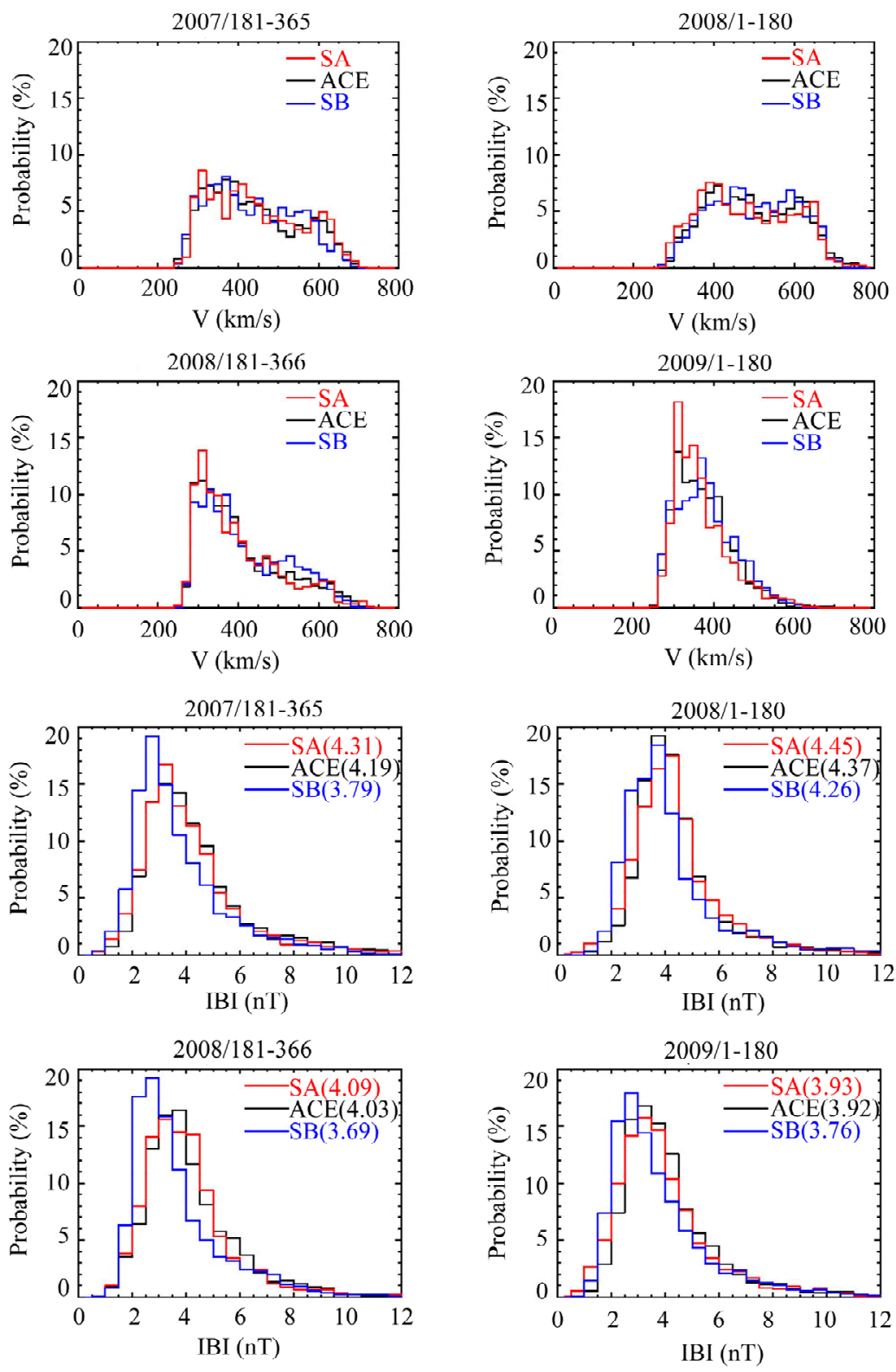

Figure 1. Histograms of 1-hour averaged solar wind velocity (four upper panels) and magnitude of interplanetary magnetic field (four lower panels) used in this study provided from ACE, STEREO-A (SA) and B (SB) spacecraft. The average magnitude of interplanetary magnetic field is shown in the parentheses.
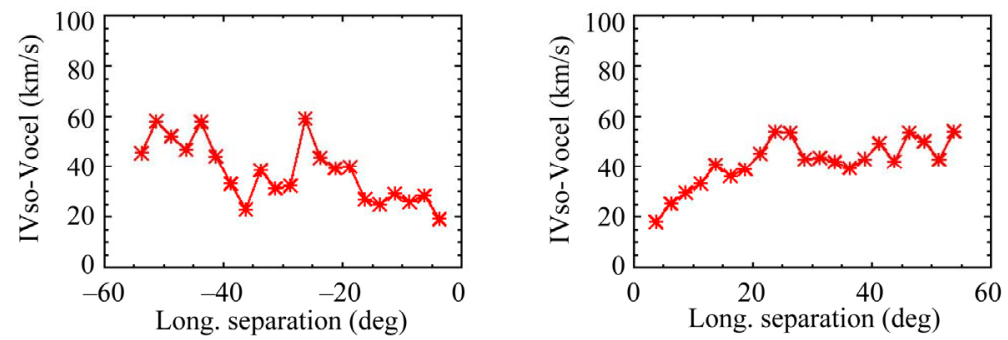

Figure 2. Difference in solar wind velocity between STEREO and ACE measurements as a function of solar longitude separation. Averages are taken over each longitude bin of 2.5 degree. The left side shows difference between STEREO-B and ACE measurements, whereas the right shows that between STEREO-A and ACE measurements. The difference is, in general, increased with the longitude separation. 
to result in geomagnetic disturbances. Similarly, dawnto-dusk component of solar wind electric field $\mathrm{V} \cdot \mathrm{Bz}$ is intruded into the terrestrial magnetosphere and drives magnetospheric convection. However, prediction of the north-south component $\mathrm{Bz}$ becomes impossible after the longitude separation is not negligible (e.g., [8]). Therefore, we use neither $\mathrm{Bz}$ nor $\mathrm{V} \cdot \mathrm{Bz}$ in this study.

The analysis period is just in the solar minimum, so that most of disturbances were due to Corotating Interaction Region (CIR) [21]. The fast solar wind stream overtakes slower streams ahead and compressed region is developed at CIRs. Richardson et al. [22] reported 70\% of geomagnetic disturbances were caused by CIRs during the solar minimum. Negative large $\mathrm{Bz}$ conditions appear as a fluctuating field line in the compressed $|\mathrm{B}|$ region of CIRs. $|\mathrm{B}|$ gives an envelope of fluctuating Bz. Therefore, we use $|\mathrm{B}|$ instead of $\mathrm{Bz}$ and, also use $\mathrm{V} \cdot|\mathrm{B}|$ instead of $\mathrm{V} \cdot \mathrm{Bz}$, in this study.

Figure 3 shows examples of relation of Kp with the three solar wind parameters measured by ACE, in which we corrected the propagation delay of the solar wind from the ACE to the earth. The red line connects averaged parameter values at each $\mathrm{Kp}$ index. We see fair correlations between $\mathrm{Kp}$ and the three solar wind parameters measured by the ACE, indicating that the three solar wind parameters are worth being predicted.

The purpose of this study is to introduce geoeffectiveness into the correlation analysis between ACE and STEREO solar wind measurements. Large Kp conditions are mostly observed during large $\mathrm{V}$, large $|\mathrm{B}|$, and/or large $\mathrm{V} \cdot|\mathrm{B}|$ conditions as shown in Figure 3. Correlation coefficient is not suitable for evaluating prediction ability in this study, since, for example, we compare the "correlation" of small V with that of large V. Correlation coefficient is significant for variables with enough variation range. Therefore, we do not treat correlation coefficient but difference of the parameter, as in Figure 2, in this study.

\section{Results}

Figure 4 shows histograms of velocity difference between STEREO-B and ACE measurements (left row) and between STEREO-A and ACE measurements (right) sorted by $\mathrm{Kp}$ index. Each histogram for a Kp range is normalized so that integration over velocity difference is $100 \%$. Color of the lines represents a $\mathrm{Kp}$ range as indicated in the figure. The distribution of velocity difference generally spreads more widely in the later period, reflecting increasing difference in solar longitude as shown in Figure 2. The distribution also tends to be narrower centered around zero for smaller Kp. During quiet intervals (e.g., $\mathrm{Kp} \leq 1$ ), the distribution is almost symmetric about zero, whereas it is asymmetric for active conditions $(\mathrm{Kp} \geq 4)$. The occasion of negative difference is much more frequent for large Kp, which means that more geomagnetic disturbances occur when we underestimate solar wind velocity at ACE. This result is quite understandable
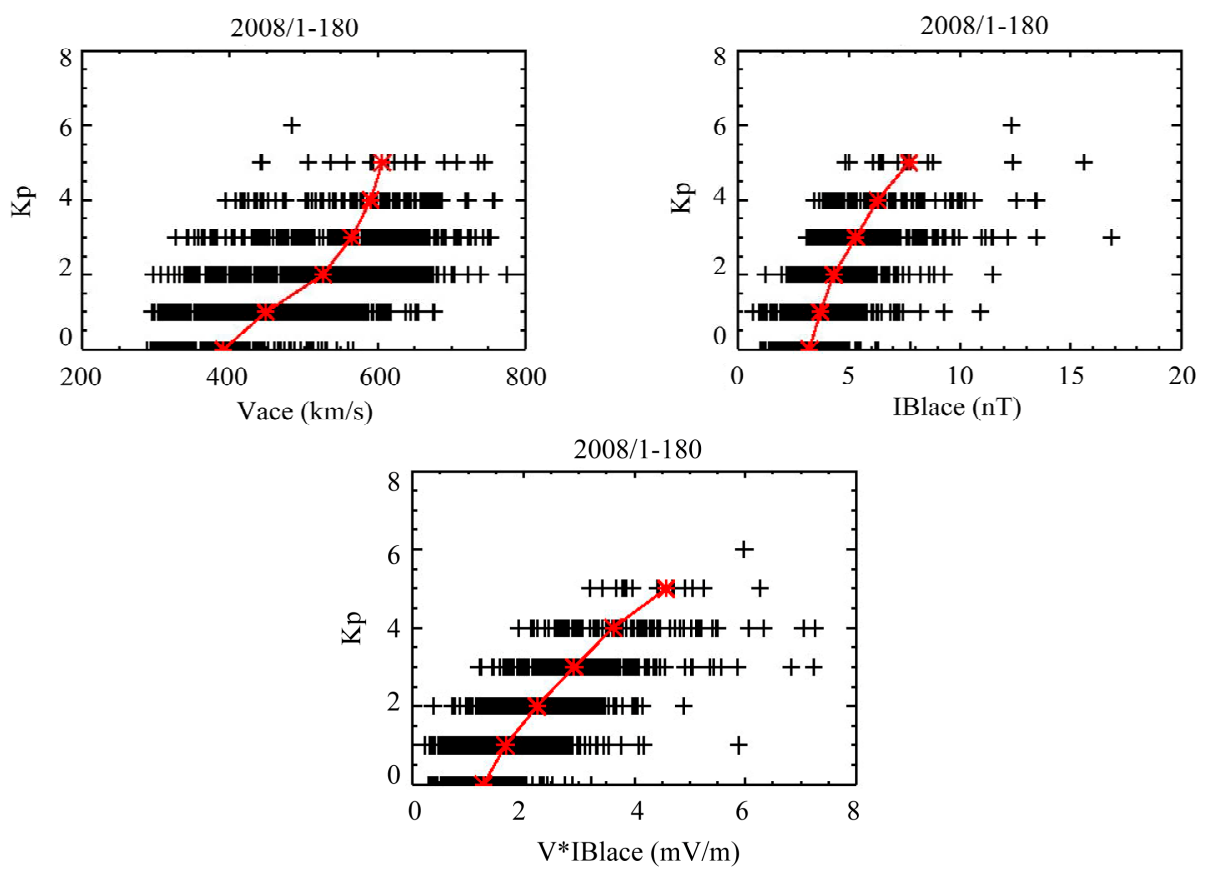

Figure 3. Examples of relation of $\mathrm{Kp}$ with solar wind velocity (upper left), magnitude of interplanetary magnetic field (upper right) and the product of these two parameters (lower left), measured at ACE. The redline connects averaged values at each Kp index. We see fair correlations between Kp and the three solar wind parameters measured by the ACE. 

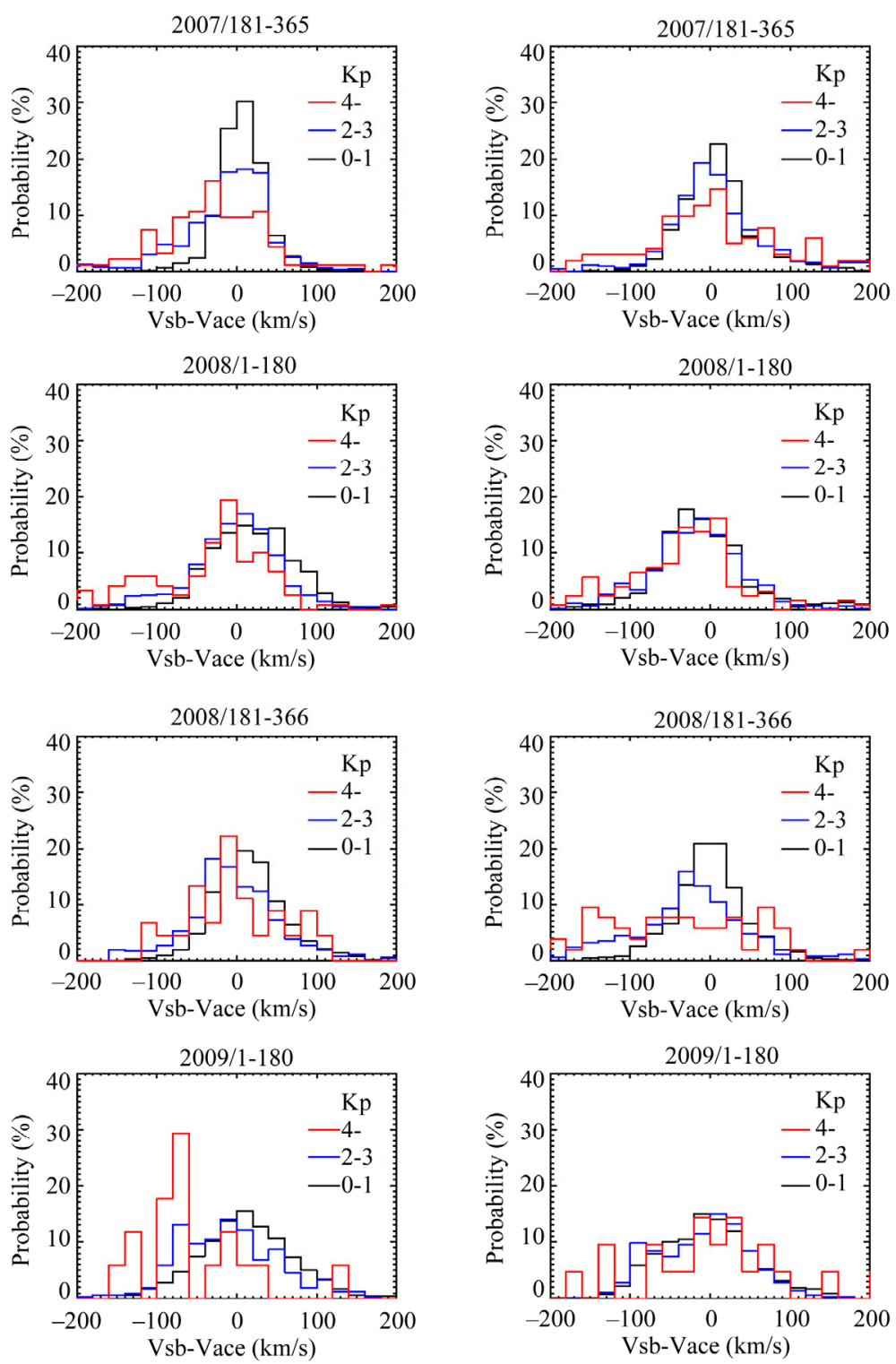

Figure 4. Histograms of velocity difference between STEREO-B and ACE measurements (left row) and between STEREO-A and ACE measurements (right) sorted by Kp index. Each histogram for a Kp range is normalized so that integration over velocity difference is $\mathbf{1 0 0} \%$. Color of the lines represents a Kp range as indicated in the figure. The occasion of negative difference is much more frequent for large $\mathrm{Kp}$, which means that more geomagnetic disturbances occur when we underestimate solar wind velocity at ACE.

since geomagnetic disturbances take place for large solar wind velocity at ACE (Figure 3(a)) and therefore overestimate seldom happens. There is no significant differrence in the distribution between estimations from STEREO-A and STEREO-B measurements.

Figures 5 and $\mathbf{6}$ show histograms of $|\mathrm{B}|$ and $\mathrm{V} \cdot|\mathrm{B}|$ differences, respectively. The format of the figures is the same as used in Figure 4. We pointed out a tendency of slightly larger $|\mathrm{B}|$ measured at STEREO-A than at ACE in Figure 1. This does not seem to affect significantly the histograms of $|\mathrm{B}|$ difference in Figure 5, though, comparing to the histograms of the left row (i.e., difference between STEREO-B and ACE), the distribution might be relatively shifted toward positive values.

Besides the slight shift of $|\mathrm{B}|$ difference, general features of the histograms in Figures $\mathbf{5}$ and $\mathbf{6}$ are similar to those found in the velocity difference. The distribution is wider in the later period due to the longer time for variation. It is also wider and more asymmetric during active intervals. The case of underestimation happens more frequently for active conditions. The large underestimation of solar wind parameters leads to large errors of predicting geomagnetic disturbances.

We quantify the underestimation of the parameters by 

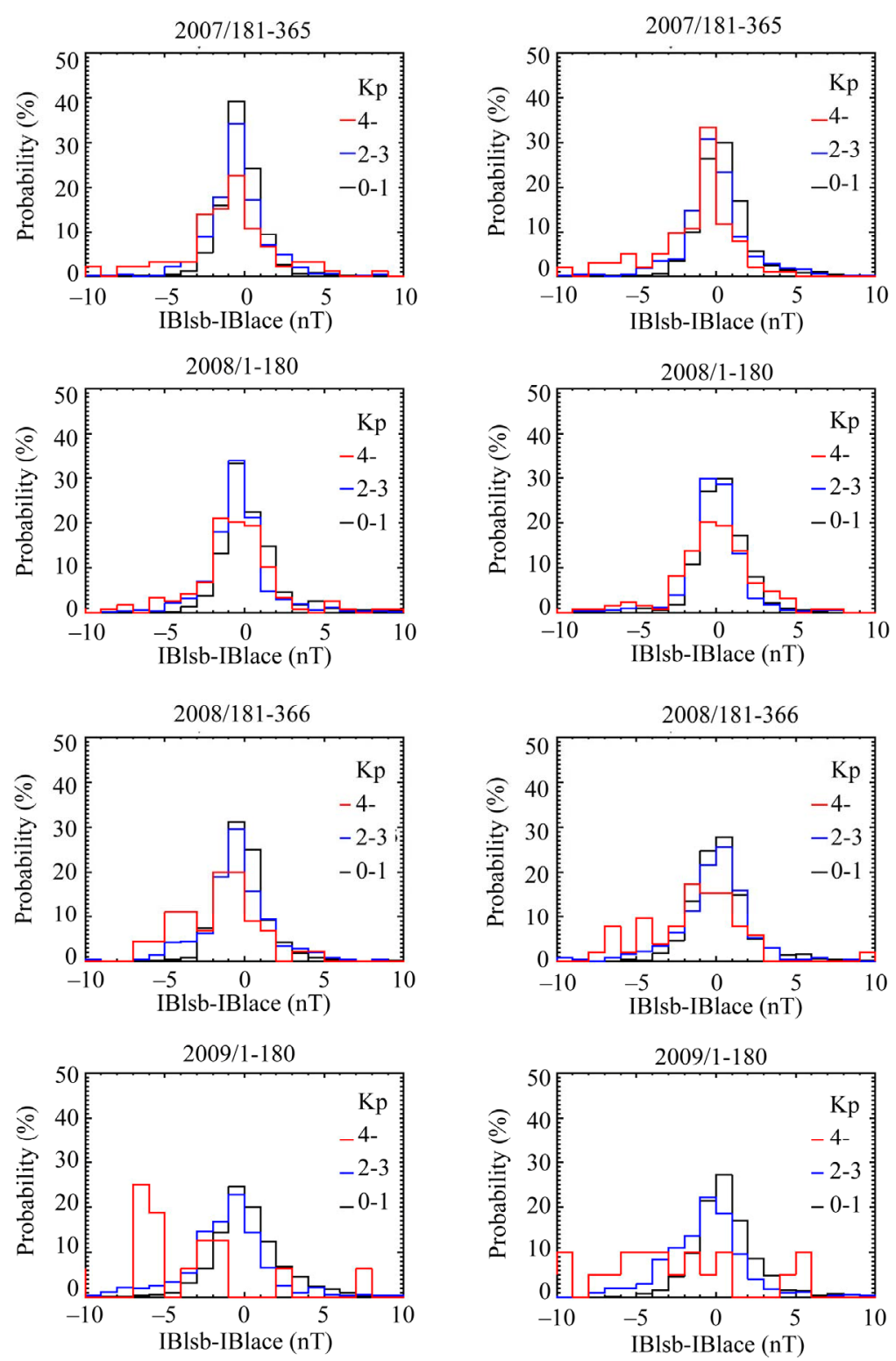

Figure 5. Histograms of $|B|$ difference between STEREO-B and ACE measurements (left row) and between STEREO-A and ACE measurements (right) sorted by Kp index. The format of the figures is the same as used in Figure 4. The distribution is wider and more asymmetric during active intervals. The case of underestimation happens more frequently for active conditions.

taking root means square (RMS) of the negative difference. We calculate the RMS of the three parameter differences sorted by the Kp index. We convert the four observation periods into solar longitude separation averaged over each period and present the RMS as a function of solar longitude separation in Figure 7. As already pointed out in Figures 4-6, the RMS is larger for active conditions and is increased with increasing longitude separation. The RMS for active conditions seems more susceptible to increasing longitude separation. There is no large difference between the RMS variations from STEREO-A and B estimations, and the RMS variation is quite symmetric about zero longitude separation.

The difference between solar wind parameters measured by STEREO and ACE causes some errors for predicting geomagnetic activities regardless of prediction method. Next we make an attempt to predict $\mathrm{Kp}$ index from actual STEREO-A and B solar wind measurements and to evaluate quantitatively its predictive abilities. There exist various methods to relate solar wind parameters with magnetospheric activities, ranging from empirical equations to numerical MHD simulation codes. Our purpose here is not to obtain any exact evaluation, but rather roughly to see whether or not the difference in 

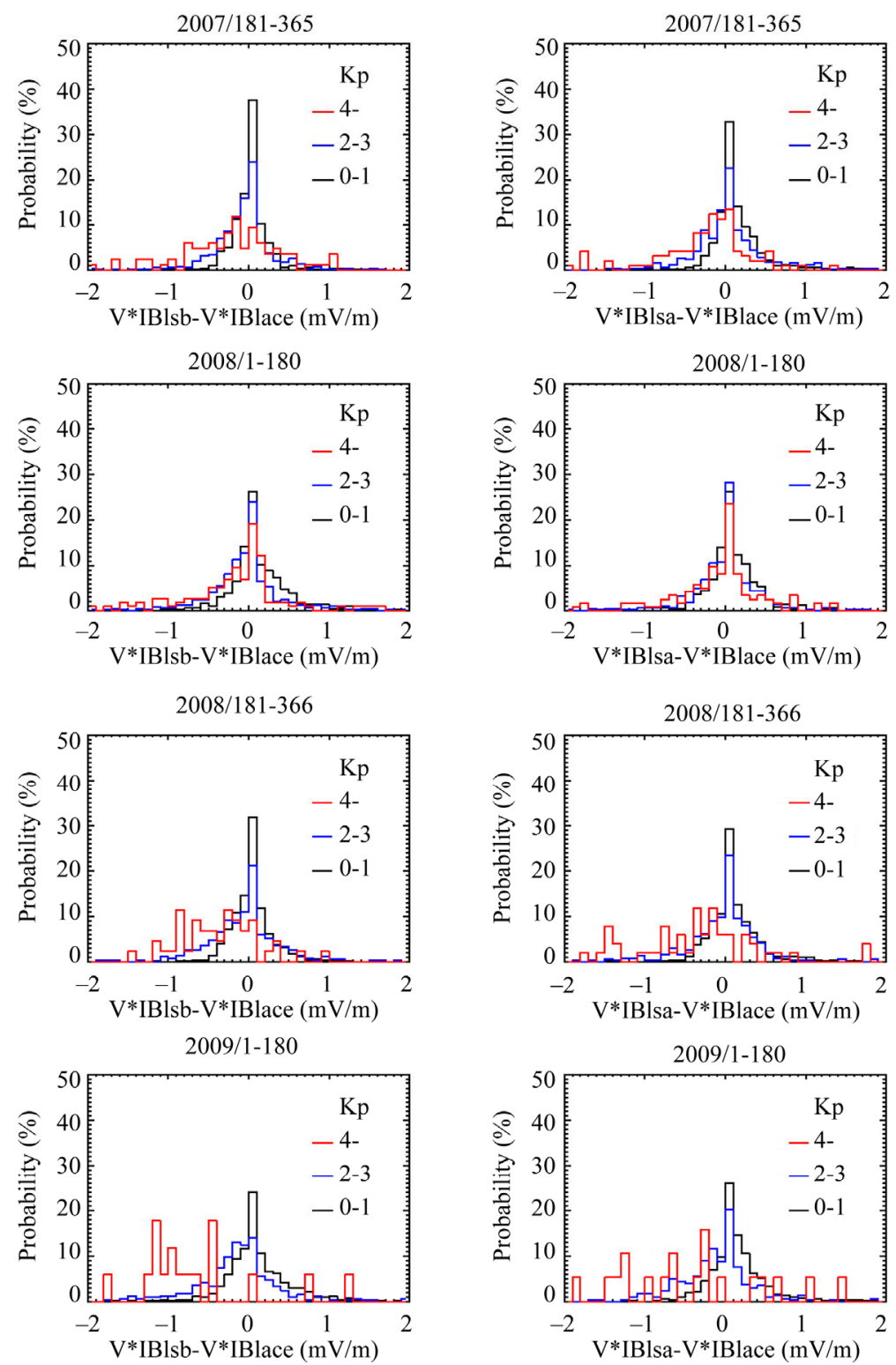

Figure 6. Histograms of $V^{*}|B|$ difference between STEREO-B and ACE measurements (left row) and between STEREO-A and ACE measurements (right) sorted by Kp index. The format of the figures is the same as used in Figure 4. The distribution is wider and more asymmetric during active intervals. The case of underestimation happens more frequently for active conditions.

solar wind parameters obtained in our analysis is significant for geomagnetic activities. For simplicity, we adopt the empirical relation of $\mathrm{Kp}$ with $\mathrm{V} \cdot|\mathrm{B}|$ for the four intervals (i.e., the red line in Figure 3(c)) to convert the difference in solar wind parameter to the Kp difference.

We take again RMS (root mean square) of the difference of the measured and predicted $\mathrm{Kp}$ indices to quantify the prediction ability. The results are summarized as a function of solar longitude separation in the left side of Figure 8 from STEREO-B and in the right from STEREO-A measurements, respectively. Color of the lines represents the same $\mathrm{Kp}$ range as used in the previous figures. The Kp difference is larger for active conditions and is increased with increasing solar longitude separation. There is no large difference between predictions from STEREO-A and B solar wind measurements. These basic features are just as expected from the results of predicting solar wind parameters (Figure 6). When the longitude separation reaches close to 50 degree, predicted $\mathrm{Kp}$ contains an error of 2 to 3 during geomagnetic disturbances $(\mathrm{Kp} \geq 4)$. On the other hand, the error remains around 1 during quiet intervals $(\mathrm{Kp} \leq 1)$ even after the longitude separation is large, so that prediction is rather successful for quiet intervals. 

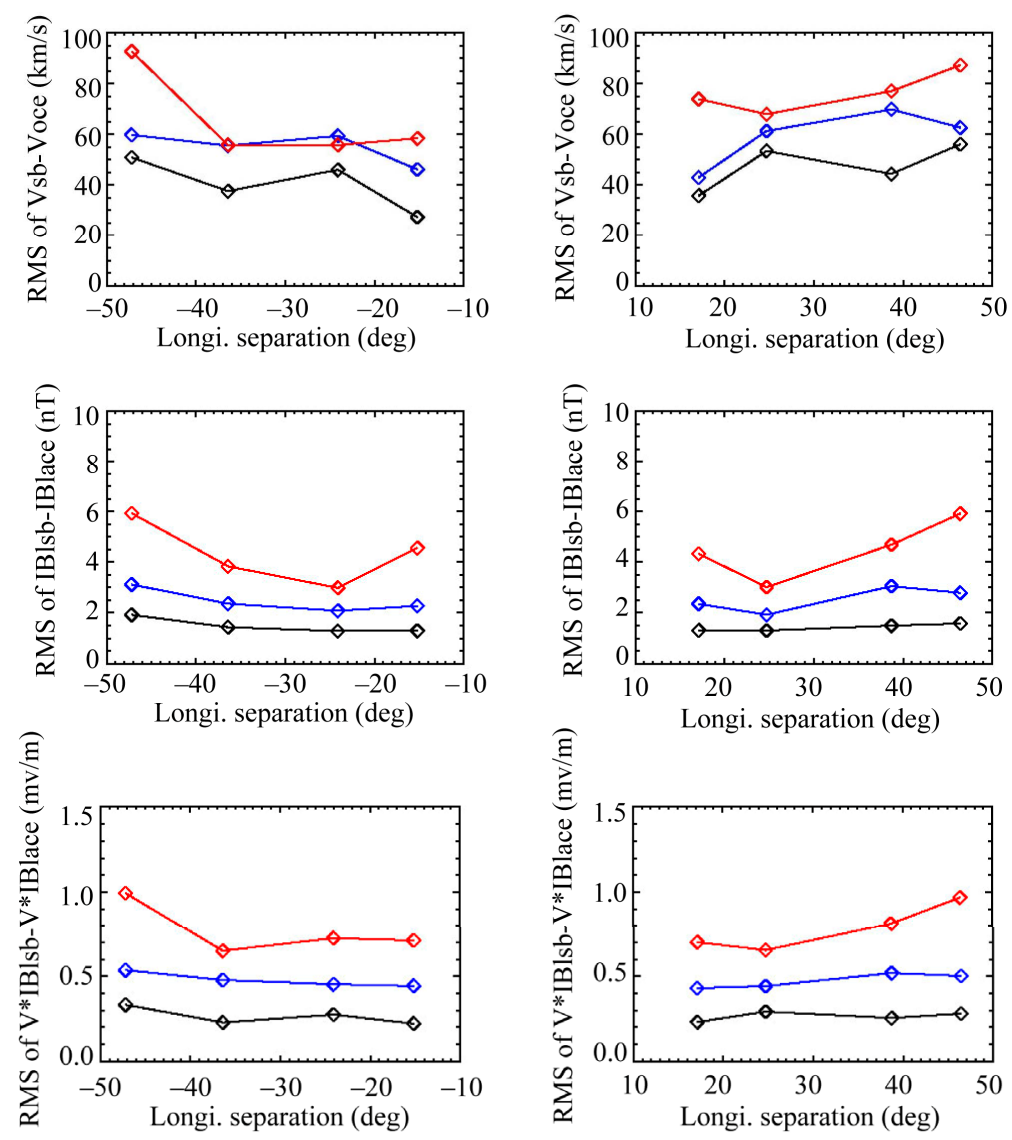

Figure 7. RMS of the three parameter differences as a function of longitude separation. The left and right sides show the RMS of the negative differences between STEREO-B and ACE and between STEREO-A and ACE measurements, respectively. Color of the lines represents the same $K p$ range (red: $K p \geq 4$, blue: $2 \leq K p \leq 3$, black: $K p \leq 1$ ) as used in the previous figures. Larger RMS is obtained at larger longitude separation and during larger Kp intervals.
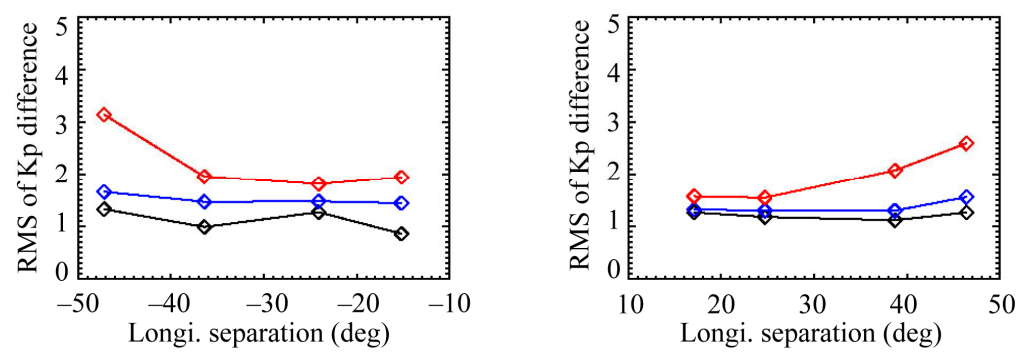

Figure 8. RMS of the predicted Kp difference as a function of longitude separation. The left and right sides show the RMS of the negative differences between STEREO-B and ACE and between STEREO-A and ACE measurements, respectively. Color of the lines represents the same $K p$ range (red: $K p \geq 4$; blue: $2 \leq K p \leq 3$; black: $K p \leq 1$ ) as used in the previous figures.

\section{Discussion}

Our results may not be so astonishing but rather easily understood qualitatively in terms of large-scale high- and low-speed solar wind structures. At 1 AU from the sun, large $\mathrm{Kp}$ intervals take place at leading edge of highspeed streams where CIR is developed. Leading edge of high-speed streams is generally short and steep, whereas trailing edge is prolonged and gradually varied. In gene- ral, it is difficult to predict exact occurrence time and interval of an event with a short duration. If the event lasts for a long time and its change is gradual, errors in prediction tend to be small.

Slow solar wind was dominant and geomagnetic activity was generally low in 2009 (e.g., [17,18]), when the longitude separation between STEREO and ACE reached its maximum in this study. We must note that the difference in the solar wind parameters for $K p \geq 4$ intervals in 
early 2009 (Figures 4-6) may be a sort of possible minimum estimation. If the solar wind is always slow, prediction is easy and error in the prediction is generally small. The difference in 2009 would be larger if the solar wind condition was similar to that in 2007 and 2008. Therefore, the predicted $\mathrm{Kp}$ difference in Figure 8 also gives a minimum evaluation of error at large longitude separation. If the solar wind had been faster in 2009, the prediction of large $\mathrm{Kp}$ interval would be more difficult at the longitude separation of 40 - 50 degree.

In this study, we deal with $\mathrm{Kp}$ index as a representative indicator for geomagnetic disturbances. There are other parameters to present magnetospheric activities and hazardous space weather conditions to be predicted such as relativistic electron flux at the geosynchronous orbit. The relativistic electron flux is correlated with $\mathrm{Kp}$ index and solar wind velocity with a delay of a few days $[23,24]$. Our results demonstrate that fast solar wind is more difficult to be predicted than slow solar wind and that large $\mathrm{Kp}$ intervals are more difficult to be predicted than small $\mathrm{Kp}$ intervals. Therefore, we expect that the same is also true for the prediction of relativistic electron flux. Our results suggest that more hazardous space weather condition, in general, is more difficult to be predicted accurately.

There are basically three factors to control the difference between STEREO and ACE solar wind measurements: temporal variation during the solar wind radial expansion, temporal variation in the solar wind source region, and latitudinal gradient in solar wind structures. The temporal variation during the solar wind radial expansion is associated with stream interactions around CIRs and is expected to be noticeable at distant regions from the sun. We found no significant difference between solar wind estimations from STEREO-A and B measurements, so that the effect of temporal variation based on stream interactions seems not largely affecting our results.

The temporal variation in the solar wind source regions can be categorized into two kinds: explosive changes by transients like CMEs and gradual changes in largescale high- and low-speed structures associated with coronal hole distribution. Our analysis period is during the solar minimum, and occurrence of CMEs is low, even if not totally negligible. There are though small-scale transients sometimes observed in slow solar wind region even around the solar minimum (e.g., [25]). These events do not exhibit significant velocity changes across the structure, but instead appear to move with the surrounding flow, so that they are not so effective to reduce the correlations of the solar wind parameters. The differences in solar wind parameters between STEREO and ACE measurements are well organized as a function of solar longitude separation. Therefore, we conclude that the occurrence of effective transients does not significantly affect our results performed on large scales.

On the other hand, the latter cause of the temporal variation (i.e., gradual changes in high- and low-speed structures) is most likely since the difference is increased with longitude separation, i.e., the lag time. Longer lag time allows more time for evolution of the high- and low-speed stream structure in the solar wind even if the variation is gradual. Opitz et al. [26] analyzed correlation of solar wind bulk velocity measured by STEREO-A and B during March-August 2007 when the longitude separation was not large, and found a decrease in correlation with increasing the lag time, which is in agreement with our results.

Large gradient in latitude is sometimes observed in interplanetary space. Rouillard et al. [27] reported a large difference between STEREO-A, B and ACE measurements after passage of a stream interface in July 2007. They attributed the solar wind difference to latitude difference of 1 degree between the spacecraft, since, based on observed EUVI image, source region of the solar wind is expected to emanate from an equatorward edge of a coronal hole where a large gradient exists. We cannot easily separate the effect of latitudinal gradient effect from that of longitude separation by means of in situ measurements in this study. Since the three spacecraft are located on the ecliptic plane, the latitude separation oscillates and its amplitude is increased with the longitude separation. Here, we rather let the latitude effect be involved into the longitude separation effect and consider the effect totally. The solar wind monitoring at separated solar longitude, for example, at the L5 point, is always accompanied with the same combined effect. We leave the subject, the discrimination of the latitude effect from the longitude lag time effect, to future studies.

Our results indicate that a simple correlation method of solar wind measurement at separated solar longitude is not enough for accurately predicting geomagnetic disturbances. The maximum longitude separation is about 50 degree during our analysis period. The longitude separation of the L5 point is 60 degree, even larger than that in this study, and the possible latitude difference of the L5 point is also larger than that suggested in this study. We should expect more difficulties in predicting geomagnetic disturbances by using in situ solar wind measurements at the L5 point. Although previous studies reported a good correlation of solar wind parameters [10,11], we should be cautious in solar wind variation. We need to develop more sophisticated prediction schemes including temporal variation effect during the solar rotation time and latitudinal gradient in the solar wind structure before solar wind monitoring at separated solar longitude in interplanetary space is installed in any practical operation system of space weather forecast. 
In conclusion, geomagnetic disturbances are more difficult to be predicted than quiet intervals. Although insitu solar wind monitoring at a vantage point trailing behind the earth would definitely improve the prediction capability of solar wind structure arriving at the earth, we emphasize that the predictive ability of geoeffective conditions would still remain low.

\section{Acknowledgements}

We thank the ACE SWEPAM and MAG instrument teams and the ACE Science Center for providing the ACE data. The STEREO PLASTIC and MAG data were obtained from the website of Space Physics Center of UCLA.

\section{REFERENCES}

[1] I. G. Richardson, E. W. Cliver and H. V. Cane, "Sources of Geomagnetic Storms for Solar Minimum and Maximum Conditions during 1972-2000," Geophysical Research Letters, Vol. 28, No. 13, 2001, pp. 2569-2572. doi:10.1029/2001GL013052

[2] R. Kataoka and Y. Miyoshi, "Flux Enhancement of Radiation Belt Electrons during Geomagnetic Storms Driven by Coronal Mass Ejections and Corotating Interaction Regions," Space Weather, Vol. 4, No. 9, 2006, pp. 1-11. doi:10.1029/2005SW000211

[3] M. Akioka, T. Nagatsuma, W. Miyake, K. Ohtaka and K. Marubashi, "The L5 Mission for Space Weather Forecasting," Advances in Space Research, Vol. 35, No. 1, 2005, pp. 65-69. doi:10.1016/j.asr.2004.09.014

[4] D. F. Webb, D. A. Biesecker, N. Gopalswamy, D. C. St. Cyr, J. M. Davila, C. J. Eyles, B. J. Thompson, K. D. C. Simunac and J. C. Johnson, "Using STEREO-B as an L5 Space Weather Pathfinder Mission," Space Research Today, Vol. 178, 2010, pp. 10-16. doi:10.1016/j.srt.2010.07.004

[5] N. Gopalswamy, J. M. Davila, O. C. St. Cyr, E. C. Sittler, F. Auchere, T. L. Duvall Jr., J. T. Hoeksema, M. Maksimovic, R. J. MaDowall, A. Szabo and M. R. Collier, "Earth-Affecting Solar Causes Observatory (EASCO): A Potential International Living with a Star Mission from Sun-Earth L5," Journal of Atmospheric and Solar-Terrestrial Physics, Vol. 73, No. 5-6, 2011, pp. 658-663. doi:10.1016/j.jastp.2011.01.013

[6] N. Gopalswamy, A. Lara, R. P. Lepping, M. L. Kaiser, D. Berdichevsky and O. C. St. Cry, "Interplanetary Acceleration of Coronal Mass Ejections," Geophysical Research Letters, Vol. 27, No. 2, 2000, pp. 145-148. doi:10.1029/1999GL003639

[7] T. Nagatsuma, M. Akioka, W. Miyake and K. Ohtaka, "Preceding Monitoring of Solar Wind toward the Earth Using STEREO," Journal of NICT, Vol. 56, No. 1-4, 2009, pp. 41-48. http://www.nict.go.jp/publication/shuppan/kihou-journal/j ourna-vol56no1_4.html

[8] P. Riley, J. Luhmann, A. Opitz, J. A. Linker and Z. Mikic,
"Interpretation of the Cross-Correlation Function of ACE and STEREO Solar Wind Velocities Using a Global MHD Model," Journal of Geophysical Research, Vol. 115, 2010, Article ID: A11104. doi:10.1029/2010JA01517

[9] D. L. Turner and X. Li, "Using Spacecraft Measurements Ahead of Earth in the Parker Spiral to Improve Terrestrial Space Weather Forecasts," Space Weather, Vol. 9, No. 1, 2011. doi:10.1029/2010SW000627

[10] W. Miyake, Y. Saito, H. Hayakawa and A. Matsuoka, "On the Correlation of the Solar Wind Observed at the L5 Point and at the Earth," Advances in Space Research, Vol. 36, No. 12, 2005, pp. 2328-2332. doi:10.1016/j.asr.2004.06.019

[11] K. D. C. Simunac, L. M. Kistler, A. B. Galvin, M. A. Popecki and C. J. Farrugia, "In Situ Observations from STEREO/PLASTIC: A Test for L5 Space Weather Monitors," Annales Geophysicae, Vol. 27, 2009, pp. 38053809. doi:10.5194/angeo-27-3805-2009

[12] A. B. Galvin, L. M. Kistler, M. A. Popecki, C. F. Farrugia, K. D. C. Simunac, L. Ellis, E. Mobius, M. A. Lee, M. Boehm, J. Carroll, A. Crawshaw, M. Conti, P. Demaine, S. Ellis, J. A. Gaidos, J. Googins, M. Granoff, A. Gustafson, D. Heirtzler, B. King, U. Knauss, J. Levasseur, S. Longworth, K. Singer, S. Turco, P. Vachon, M. Vosbury, M. Widholm, L. M. Blush, R. Karrer, P. Bochsler, H. Daoudi, A. Etter, J. Fisher, J. Jost, A. Opitz, M. Sigrist, P. Wurz, B. Klecker, M. Ertl, E. Seidenschwang, R. F. Wimmer-Schweingruber, M. Koeten, B. Thompson and D. Steinfeld, "The Plasma and Suprathermal Ion Composition (PLASTIC) Investigation on the STEREO Observatories," Space Science Reviews, Vol. 136, No. 1-4, 2008, pp. 437-486. doi:10.1007/s11214-007-9296-X

[13] M. H. Acuna, D. Curtis, J. L. Scheifele, C. T. Russell, A. Szabo and J. G. Luhman, "The STEREO/IMPACT Magnetic Field Experiment," Space Science Reviews, Vol. 136, No. 1-4, 2008, pp. 203-226. doi:10.1007/s11214-007-9259-2

[14] D. J. McComas, S. J. Bame, P. Barker, W. C. Feldman, J. L. Phillips, P. Riley and J. W. Griffee, "Solar Wind Electron Proton Alpha Monitor (SWEPAM) for the Advanced Composition Explorer," Space Science Reviews, Vol. 86, No. 1-4, 1998, pp. 563-612. doi:10.1023/A:1005040232597

[15] C. W. Smith, J. L'Heureux, N. S. Ness, M. H. Acuna, L. F. Burlaga and J. Scheifele, "The ACE Magnetic Field Experiment," Space Science Reviews, Vol. 86, No. 1-4, 1998, pp. 613-632. doi:10.1023/A:1005092216668

[16] R. L. Mcpherron, "Magetospheric Dynamics," In: M. Kivelson and C. T. Russell, Eds., Introduction to Space Physics, Cambridge University Press, Cambridge, 1995, pp. 400-458.

[17] R. Kataoka and Y. Miyoshi, "Why Are Relativistic Electron Persistently Quiet at Geosynchronous Orbit in 2009?" Space Weather, Vol. 8, 2010, Article ID: S08002. doi:10.1029/2010SW000571

[18] C. T. Russell, J. G. Luhmann and L. K. Jian, "How Unprecedented a Solar Minimum?" Reviews of Geophysics, Vol. 48, No. RG2004, 2010. doi:10.1029/2009RG000316 
[19] W. Miyake, T. Mukai, K.-I. Oyama, T. Terasawa, K. Hirao and A. J. Lazarus, "Thin Equatorial Low Speed Region in the Solar Wind Observed during the Recent Solar Minimum," Journal of Geophysical Research, Vol. 94, No. A11, 1989, pp. 15359-15365. doi:10.1029/JA094iA11p15359

[20] D. J. McComas, P. Riley, J. T. Gosling, A. Balogh and R. Forsyth, "Ulysses' Rapid Crossing of the Polar Coronal Hole Boundary," Journal of Geophysical Research, Vol. 103, No. A2, 1998, pp. 1955-1967. doi:10.1029/97JA01459

[21] V. Pizzo, "A Three-Dimensional Model of Corotating Streams in the Solar Wind, 1. Theoretical Foundations," Journal of Geophysical Research, Vol. 83, No. A12, 1978, pp. 5563-5572. doi:10.1029/JA083iA12p05563

[22] I. G. Richardson, E. W. Cliver and H. V. Cane, "Sources of Geomagnetic Activity over the Solar Cycle: Relative Importance of Coronal Mass Ejections, High-Speed Streams, and Slow Solar Wind," Journal of Geophysical Research, Vol. 105, No. A8, 2000, pp. 18203-18213. doi:10.1029/1999JA000400

[23] T. Nagai, "'Space Weather Forecast': Prediction of Relativistic Electron Intensity of Synchronous Orbit," Geophysical Research Letters, Vol. 15, No. 5, 1988, pp. 425428. doi:10.1029/GL015i005p00425

[24] D. N. Baker, R. L. McPherron, T. E. Cayton and R. W. Klebesadel, "Linear Prediction Filter Analysis of Relativistic Electron Properties at 6.6 R $\mathrm{E}$," Journal of Geophysi- cal Research, Vol. 95, No. A9, 1999, pp. 15133-15140. doi:10.1029/JA095iA09p15133

[25] E. J. Kilpua, J. G. Luhmann, J. T. Gosling, Y. Li, H. Elliott, C. T. Russell, L. Jian, A. B. Galvin, D. Larson, P. Schroeder, K. Simunac and G. Petrie, "Small Solar Wind Transients and Their Connection to the Large-Scale Coronal Structure," Solar Physics, Vol. 256, No. 1-2, 2009, pp. 327-344. doi:10.1007/s11207-009-9366-1

[26] A. Opitz, A. Karrer, P. Wurz, A. B. Galvin, P. Bochsler, M. L. Blush, H. Daoudi, L. Ellis, C. J. Farrugia, C. Giammanco, L. M. Kistler, B. Klecker, H. Kucharek, M. A. Lee, E. Möbius, M. Popecki, M. Sigrist, K. Simunac, K. Singer, B. Thompson and R. F. Wimmer-Schweingruber, "Temporal Evolution of the Solar Wind Bulk Velocity at Solar Minimum by Correlating the STEREO A and B PLASTIC Measurements," Solar Physics, Vol. 256, No. 1-2, 2009, pp. 365-377. doi:10.1007/s11207-008-9304-7

[27] A. P. Rouillard, N. P. Savani, J. A. Davies, B. Lavraud, R. J. Forsyth, S. K. Morley, A. Opitz, N. R. Sheeley, L. F. Burlaga, J.-A. Sauvaud, K. D. C. Simunac, J. G. Luhmann, A. B. Galvin, S. R. Crothers, C. J. Davis, R. A. Harrison, M. Lockwood, C. J. Eyles, D. Bewsher and D. S. Brown, "A Multispacecraft Analysis of a Small-Scale Transient Entrained by Solar Wind Streams," Solar Physics, Vol. 256, No. 1-2, 2009, pp. 307-326. doi:10.1007/s11207-009-9329-6 